\title{
THE REAL HYPERSURFACE OF TYPE (B) WITH TWO DISTINCT PRINCIPAL CURVATURES IN A COMPLEX HYPERBOLIC SPACE
}

\author{
KATSUFumi YAmashita AND SADAhiro MAEDA
}

\begin{abstract}
Real hypersurfaces $M^{2 n-1}$ of type (B) in $\mathbf{C} H^{n}(c), n \geqq 2$ are known as interesting examples of Hopf hypersurfaces with constant principal curvatures. They are homogeneous in this ambient space. Moreover, the numbers of distinct principal curvatures of all real hypersurfaces of type (B) with radius $r \neq(1 / \sqrt{|c|}) \log _{e}(2+\sqrt{3})$ are 3 . When $r=(1 / \sqrt{|c|}) \log _{e}(2+\sqrt{3})$, the real hypersurface of type (B) has two distinct principal curvatures. The purpose of this paper is to characterize this Hopf hypersurface having two distinct constant principal curvatures.
\end{abstract}

\section{Introduction}

J. Berndt and H. Tamaru ([4]) classified all homogeneous real hypersurfaces $M^{2 n-1}$ in a complex $n(\geqq 2)$-dimensional complex hyperbolic space $\mathbf{C} H^{n}(c)$ of constant holomorphic sectional curvature $c(<0)$, namely these real hypersurfaces are orbits of some subgroups of the full isometry group $\mathrm{I}\left(\mathrm{CH}^{n}(c)\right)$ of the ambient space $\mathbf{C} H^{n}(c)$. Note that they are not necessarily Hopf hypersurfaces (for the definition and fundamental properties of Hopf hypersurfaces, see section 2). There exist many homogeneous non-Hopf hypersurfaces as well as many homogeneous Hopf hypersurfaces in this space. On the contrary every homogeneous real hypersurface in a complex $n(\geqq 2)$-dimensional complex projective space $\mathbf{C} P^{n}(c)$ of constant holomorphic sectional curvature $c(>0)$ is a Hopf hypersurface (cf. [11]).

It is well-known that there exist no totally umbilic real hypersurfaces of $\mathbf{C} H^{n}(c)(n \geqq 2)$. In this context, we recall that in $\mathbf{C} H^{n}(c)(n \geqq 3)$, a connected real hypersurface $M$ has at most two distinct principal curvatures at each point of $M$ if and only if $M$ is locally congruent to either a geodesic sphere $G(r)$ of radius $r(0<r<\infty)$ in $\mathbf{C} H^{n}(c)$, a tube of radius $r(0<r<\infty)$ over a complex

2000 Mathematics Subject Classification. Primary 53C40, Secondary 53C22.

Key words and phrases. real hypersurfaces of type (B), complex hyperbolic spaces, the holomorphic distribution, restricted principal distributions, the contact form, geodesics, circles.

The second author is partially supported by Grant-in-Aid for Scientific Research (C) (No. 23540097), Japan Society for the Promotion of Sciences.

Received October 2, 2012; revised April 18, 2013. 
hyperplane $\mathbf{C} H^{n-1}(c)$, a horosphere or the real hypersurface of type (B) with two distinct principal curvatures (see [8]). Here, all of these real hypersurfaces are homogeneous in $\mathrm{CH}^{n}(c)$.

A tube of radius $r(0<r<\infty)$ around a totally real totally geodesic $\mathbf{R} H^{n}(c / 4)$ of constant sectional curvature $c / 4$ is called a real hypersuface of type (B) in $\mathbf{C} H^{n}(c)$. When $r \neq(1 / \sqrt{|c|}) \log _{e}(2+\sqrt{3})$, every real hypersurface $M$ of type (B) has three distinct constant principal curvatures $\lambda_{1}=(\sqrt{|c|} / 2) \operatorname{coth}(\sqrt{|c|} r / 2)$, $\lambda_{2}=(\sqrt{|c|} / 2) \tanh (\sqrt{|c|} r / 2)$ and $\delta=\sqrt{|c|} \tanh (\sqrt{|c|} r)$ with $A \xi=\delta \xi$, where $A$ and $\xi$ are the shape operator and the characteristic vector of $M$, respectively (see section 2). The real hypersurface of type (B) with radius $r=$ $(1 / \sqrt{|c|}) \log _{e}(2+\sqrt{3})$ has two distinct constant principal curvatures $\lambda_{1}=\delta=$ $\sqrt{3|c|} / 2$ and $\lambda_{2}=\sqrt{|c|} /(2 \sqrt{3})$ (cf. [3]).

The purpose of this paper is to characterize the real hypersurface $M$ of type (B) with two distinct principal curvatures in $\mathbf{C} H^{n}(c)$ in terms of the derivative of its shape operator $A$, the exterior differentiation $d \eta$ of its contact form $\eta$ and the extrinsic shape of some geodesics of $M$ (see Theorem). Our motivation is based on the following three facts on real hypersurfaces $M$ of $\mathbf{C H}^{n}(c)$ :

(1) There exist no real hypersurfaces $M$ with parallel shape operator $A$ (see $[7,9])$;

(2) There exist no real hypersurfaces $M$ with closed contact form $\eta$, namely $d \eta \neq 0$ on $M$ (see [1]);

(3) There exist no real hypersurfaces $M$ all of whose geodesics are mapped to circles in the ambient space $\mathbf{C H}^{n}(c)$ (see [10]).

We remark that a characterization of real hypersurfaces $M$ of type (B) having two distinct principal curvatures in $\mathbf{C} H^{n}(c)$ was already given by Conditions (1), (2) in Lemma $\mathrm{C}$ and Condition $3_{c}$ ) in our Theorem, which are different from the above properties (1) and (2) (see [6]).

In this paper, we characterize the real hypersurface $M$ of type (B) with two distinct principal curvatures in $\mathbf{C} H^{n}(c)$ from the above properties (1), (2) and (3).

The authors would like to express their hearty thanks to the referee for improving this paper.

\section{Preliminaries}

Let $M^{2 n-1}$ be a real hypersurface of $\mathbf{C} H^{n}(c), n \geqq 2$ and $\mathscr{N}$ a unit local normal vector field on $M$. The Riemannian connections $\tilde{\nabla}$ of $\mathbf{C} H^{n}(c)$ and $\nabla$ of $M$ are related by the following formulas of Gauss and Weingarten:

$$
\begin{gathered}
\tilde{\nabla}_{X} Y=\nabla_{X} Y+g(A X, Y) \mathscr{N}, \\
\tilde{\nabla}_{X} \mathscr{N}=-A X
\end{gathered}
$$

for any vector fields $X$ and $Y$ on $M$, where $g$ is the Riemannian metric of $M$ induced from the standard metric $G$ of the ambient space $\mathbf{C} H^{n}(c)$ and $A$ is the shape operator of $M$ in $\mathbf{C} H^{n}(c)$. An eigenvector $X$ of the shape operator $A$ is called a principal curvature vector and an eigenvalue $\lambda$ of $A$ is called a principal 
curvature. We call $V_{\lambda}:=\{X \in T M \mid A X=\lambda X\}$ a principal distribution associated with principal curvature $\lambda$.

It is known that $M$ has an almost contact metric structure induced from the Kähler structure $J$ of $\mathbf{C} H^{n}(c)$, namely we have a quartet $(\phi, \xi, \eta, g)$ defined by

$$
g(\phi X, Y)=G(J X, Y), \quad \xi=-J \mathscr{N} \quad \text { and } \quad \eta(X)=g(\xi, X)=G(J X, \mathscr{N})
$$

which satisfy

$$
\phi^{2} X=-X+\eta(X) \xi, \quad g(\xi, \xi)=1, \quad \phi \xi=0 .
$$

It follows from (2.1), (2.2) and $\tilde{\nabla} J=0$ that

$$
\begin{gathered}
\left(\nabla_{X} \phi\right) Y=\eta(Y) A X-g(A X, Y) \xi, \\
\nabla_{X} \xi=\phi A X .
\end{gathered}
$$

In this paper, we call $V_{\lambda}^{0}:=\{X \in T M \mid A X=\lambda X, X \perp \xi\}$ a restricted principal distribution associated with principal curvature $\lambda$.

For later use we prepare the following Codazzi equation of $M$ in $\mathbf{C H}^{n}(c)$ :

$$
\left(\nabla_{X} A\right) Y-\left(\nabla_{Y} A\right) X=(c / 4)\{\eta(X) \phi Y-\eta(Y) \phi X-2 g(\phi X, Y) \xi\} .
$$

A real hypersurface $M$ of $\mathbf{C H}(c)$ is a Hopf hypersurface if the characteristic vector $\xi$ is a principal curvature vector at its each point. In the following, for a Hopf hypersurface $M$ we set $A \xi=\delta \xi$ on $M$. It is known that a tube of sufficiently small constant radius around every Kähler submanifold of $\mathbf{C} H^{n}(c)$ is a Hopf hypersurface. We review the following lemma which is a useful tool in the theory of Hopf hypersurfaces in $\mathbf{C} H^{n}(c), n \geqq 2$ (cf. [7, 9]).

Lemma A. For a Hopf hypersurface $M^{2 n-1}(n \geqq 2)$ with principal curvature $\delta$ corresponding to the characteristic vector field $\xi$ in $\mathbf{C} H^{n}(c)$, we have the following.

(1) $\delta$ is constant locally on $M$.

(2) If $X$ is a tangent vector of $M$ perpendicular to $\xi$ with $A X=\lambda X$, then $(2 \lambda-\delta) A \phi X=(\delta \lambda+(c / 2)) \phi X$.

Remark 1. A horosphere of $\mathbf{C} H^{n}(c)$ shows that we must consider the case of $2 \lambda-\delta=\delta \lambda+(c / 2)=0$ in the case of $c<0$.

We next recall the following property of the holomorphic distribution $T^{0} M=\{X \in T M \mid X \perp \xi\}$ of a Hopf hypersurface $M$ in $\mathbf{C} H^{n}(c)$ (see [5]).

Lemma B. The holomorphic distribution $T^{0} M=\{X \in T M \mid X \perp \xi\}$ of every Hopf hypersurace $M$ in $\mathbf{C} H^{n}(c), n \geqq 2$ is not integrable.

In this context, it is natural to consider a problem that does there exist a Hopf hypersurface $M$ in $\tilde{M}_{n}(c)$ satisfying that $T^{0} M$ is decomposed as the direct sum of integrable distributions? The following lemma gives a characterization of all real hypersurfaces of type (B) from this viewpoint $([5,6])$. 
Lemma C. A connected real hypersurface $M^{2 n-1}$ (with Riemannian connection $\nabla)$ of $\mathbf{C} H^{n}(c), n \geqq 2$ is of type $(B)$ if and only if $M$ satisfies the following two conditions:

(1) The holomorphic distribution $T^{0} M$ of $M$ is decomposed as the direct sum of restricted principal distributions $V_{\lambda_{i}}^{0}=\left\{X \in T^{0} M \mid A X=\lambda_{i} X\right\}$;

(2) Every $V_{\lambda_{i}}^{0}$ in Condition (1) is integrable and each of its leaves is a totally geodesic submanifold of $M$, namely $\nabla_{X} Y \in V_{\lambda_{i}}^{0}$ for all vectors $X$ and $Y$ of any $V_{\lambda_{i}}^{0}$.

We here recall the following classification theorem of Hopf hypersurfaces with constant principal curvatures in $\mathbf{C} H^{n}(c)$, which is due to Berndt ([3]).

THEOREM A. Let $M^{2 n-1}$ be a connected Hopf hypersurface all of whose principal curvatures are constant in $\mathbf{C} H^{n}(c), n \geqq 2$. Then $M$ is locally congruent to one of the following:

$\left(\mathrm{A}_{0}\right) A$ horosphere in $\mathbf{C} H^{n}(c)$;

$\left(\mathrm{A}_{1,0}\right)$ A geodesic sphere $G(r)$ of radius $r(0<r<\infty)$;

$\left(\mathrm{A}_{1,1}\right)$ A tube of radius $r(0<r<\infty)$ around a totally geodesic $\mathbf{C H}^{n-1}(c)$;

$\left(\mathrm{A}_{2}\right)$ A tube of radius $r(0<r<\infty)$ around a totally geodesic $\mathbf{C H}^{\ell}(c)$ $(1 \leqq \ell \leqq n-2)$;

(B) A tube of radius $r(0<r<\infty)$ around a totally real totally geodesic $\mathbf{R} H^{n}(c / 4)$.

These real hypersurfaces are said to be of types $\left(A_{0}\right),\left(A_{1}\right),\left(A_{1}\right),\left(A_{2}\right)$ and $(B)$. Here, type $\left(A_{1}\right)$ means either type $\left(A_{1,0}\right)$ or type $\left(A_{1,1}\right)$. Unifying real hypersurfaces of type $\left(A_{0}\right),\left(A_{1}\right)$ and $\left(A_{2}\right)$, we call them real hypersurfaces of type $(A)$. Except the real hypersurface of type $(\mathrm{B})$ with radius $r=(1 / \sqrt{|c|}) \log _{e}(2+\sqrt{3})$, the numbers of distinct principal curvatures of these real hypersurfaces are $2,2,2,3,3$, respectively (for details, see [3]).

At the end of this section we review the definition of circles in Riemannian geometry. A real smooth curve $\gamma=\gamma(s)$ parameterized by its arclength $s$ in a Riemannian manifold $M$ with Riemannian connection $\nabla$ is called a circle of curvature $k$ if it satisfies the ordinary differential equations $\nabla_{\dot{\gamma}} \dot{\gamma}=k Y_{s}, \nabla_{\dot{\gamma}} Y_{s}=$ $-k \dot{\gamma}$ with a field $Y_{s}$ of unit vectors along $\gamma$. Here $k(\geqq 0)$ is constant and $Y_{s}$ is called the unit principal normal vector of $\gamma$. A circle of null curvature is nothing but a geodesic. The definition of a circle is equivalent to saying that it is a curve $\gamma=\gamma(s)$ on $M$ with Riemannian metric $g$ satisfying the ordinary differential equation

$$
\nabla_{\dot{\gamma}}\left(\nabla_{\dot{\gamma}} \dot{\gamma}\right)+g\left(\nabla_{\dot{\gamma}} \dot{\gamma}, \nabla_{\dot{\gamma}} \dot{\gamma}\right) \dot{\gamma}=0
$$

\section{Statements of results}

THEOREM. Let $M^{2 n-1}$ be a connected real hypersurface of $\mathbf{C} H^{n}(c), n \geqq 2$. Then the following conditions (1), (2) and (3) are mutually equivalent. 
(1) $M$ is locally congruent to the real hypersurface of type (B) with two distinct principal curvatures in $\mathbf{C H}^{n}(c)$.

(2) $M$ satisfies the following two conditions $2_{a}$ ) and $2_{b}$ ).

$2_{a}$ ) The exterior differentiation $d \eta$ of the contact form $\eta$ on $M$ which is given by $d \eta(X, Y):=(1 / 2)\{X(\eta(Y))-Y(\eta(X))-\eta([X, Y])\}$ holds either $d \eta(X, Y)=(\sqrt{|c| / 3}) g(X, \phi Y)$ for all $X, Y \in T M$ or $d \eta(X, Y)=(-\sqrt{|c| / 3}) g(X, \phi Y)$ for all $X, Y \in T M$.

$\left.2_{b}\right)$ There exist two geodesics $\gamma_{i}=\gamma_{i}(s)(i=1,2)$ on $M$ through a point $x=\gamma_{1}(0)=\gamma_{2}(0)$ with initial vectors $\dot{\gamma}_{i}(0)$ orthogonal to $\xi_{\gamma_{i}(0)}$ which are mapped to circles of different positive curvatures.

(3) $M$ satisfies the following three conditions $3_{a}$ ), $3_{b}$ ) and $3_{c}$ ).

$3_{a}$ ) The holomorphic distribution $T^{0} M$ of $M$ is decomposed as the direct sum of restricted principal distributions $V_{\lambda_{i}}^{0}=\left\{X \in T^{0} M \mid A X=\lambda_{i} X\right\}$.

$3_{b}$ ) The derivative of the shape operator $A$ of $M$ satisfies $\left(\nabla_{X} A\right) Y=0$ for all vectors $X, Y$ of each $V_{\lambda_{i}}^{0}$ in $3_{a}$ ).

$3_{c}$ ) There exist two geodesics $\gamma_{i}=\gamma_{i}(s)(i=1,2)$ on $M$ through a point $x=\gamma_{1}(0)=\gamma_{2}(0)$ with initial vectors $\dot{\gamma}_{i}(0)$ orthogonal to $\xi_{\gamma_{i}(0)}$ which are mapped to circles of positive curvatures $3 k$ and $k$, respectively.

Proof. We first prove that Condition (1) implies both Conditions (2) and (3). Let $M$ be the real hypersurface of type (B) with two distinct principal curvatures. Then our real hypersurface $M$ satisfies (see Lemma A(2))

$$
A \xi=\lambda_{1} \xi, \quad T^{0} M=V_{\lambda_{1}}^{0} \oplus V_{\lambda_{2}}^{0} \quad \text { and } \quad \phi V_{\lambda_{1}}^{0}=V_{\lambda_{2}}^{0}
$$

where $\lambda_{1}:=\sqrt{3|c|} / 2$ and $\lambda_{2}=\sqrt{|c|} /(2 \sqrt{3})$. Hence we easily see that

$$
(\phi A+A \phi) X=\left(\lambda_{1}+\lambda_{2}\right) \phi X \text { for all } X \in T M .
$$

It follows from (2.5) and (3.1) that

$$
\begin{aligned}
d \eta(X, Y) & =\frac{1}{2}\left(g\left(Y, \nabla_{X} \xi\right)-g\left(X, \nabla_{Y} \xi\right)\right) \\
& =\frac{1}{2} g((\phi A+A \phi) X, Y)=\frac{\sqrt{|c|}}{\sqrt{3}} g(\phi X, Y) \\
& =-\frac{\sqrt{|c|}}{\sqrt{3}} g(X, \phi Y)
\end{aligned}
$$

Needless to say, when we take a unit normal vector $\mathscr{N}$ with $A \xi=-\lambda_{1} \xi$, we get $d \eta(X, Y)=(\sqrt{|c|} / \sqrt{3}) g(X, \phi Y)$ for all $X, Y \in T M$.

We next take two geodesics $\gamma_{1}=\gamma_{1}(s)$ and $\gamma_{2}=\gamma_{2}(s)$ on $M$ through an arbitrary fixed point $x=\gamma_{1}(0)=\gamma_{2}(0)$ with initial vectors $\dot{\gamma}_{1}(0) \in V_{\lambda_{1}}^{0}$ and $\dot{\gamma}_{2}(0) \in V_{\lambda_{2}}^{0}$, respectively. Then by virtue of Lemma $\mathrm{C}$ we find that these curves $\gamma_{1}$ and $\gamma_{2}$ can be considered as geodesics on some leaves $L_{\lambda_{1}}$ and $L_{\lambda_{2}}$ of the restricted principal distributions $V_{\lambda_{1}}^{0}$ and $V_{\lambda_{2}}^{0}$, respectively. We here explain these leaves $L_{\lambda_{1}}$ and $L_{\lambda_{2}}$ in detail. Due to (2.1) we see that the leaves $L_{\lambda_{1}}$ and $L_{\lambda_{2}}$ are totally umbilic hypersurfaces of an $n$-dimensional totally real totally geodesic 
real hyperbolic space $\mathbf{R} H^{n}(c / 4)$ of constant sectional curvature $c / 4$ in $\mathbf{C} H^{n}(c)$. Hence these leaves are locally congruent to real space forms $M^{n-1}\left(d_{i}\right)$ of constant sectional curvatures $d_{i}(i=1,2)$, respectively. So we have the equations $d_{i}-(c / 4)=\lambda_{i}^{2}$. Thus we know that $L_{\lambda_{1}}$ and $L_{\lambda_{2}}$ are locally congruent to real space forms $M^{n-1}(|c| / 2)$ of constant sectional curvature $|c| / 2$ and $M^{n-1}(c / 6)$ of constant sectional curvature $c / 6$, respectively. This, together with Equation (2.1), shows that these geodesics $\gamma_{1}$ and $\gamma_{2}$ are mapped to circles of different positive curvatures $\lambda_{1}$ and $\lambda_{2}$ in $\mathbf{C} H^{n}(c)$, respectively. Thus we obtain Condition (2).

The above discussion yields that Conditions $3_{a}$ ) and $3_{c}$ ) are immediate consequences of Condition (1). So we here verify Condition $3_{b}$ ). Thanks to Lemma $\mathrm{C}$ our real hypersurface $M$ satisfies $\nabla_{X} Y \in V_{\lambda_{i}}^{0}$ for all $X, Y$ of the restricted principal foliation $V_{\lambda_{i}}^{0}(i=1,2)$. For all $X, Y \in V_{\lambda_{i}}^{0}(i=1,2)$ with $\lambda_{1}=\sqrt{3|c|} / 2$ and $\lambda_{2}=\sqrt{|c|} /(2 \sqrt{3})$, we have

$$
\begin{aligned}
\left(\nabla_{X} A\right) Y & =\nabla_{X}(A Y)-A \nabla_{X} Y \\
& =\lambda_{i} \nabla_{X} Y-\lambda_{i} \nabla_{X} Y=0,
\end{aligned}
$$

so that we get Condition $3_{b}$ ). Therefore we can see that Condition (1) implies both Conditions (2) and (3).

Conversely, we suppose Condition (2). Then, from Condition $2_{a}$ ) and the above discussion we can take a unit normal vector $\mathscr{N}$ on $M$ satisfying (3.1). Setting $X=\xi$ in (3.1), we get $\phi A \xi=0$, so that our real hypersurface $M$ is a Hopf hypersurface. Next we take a principal curvature vector field $X(\perp \xi)$ with principal curvature $\lambda$ in (3.1). Suppose that $2 \lambda-\delta \neq 0$ at some point $x \in M$. Hence, from the continuity of the function $2 \lambda-\delta$ there exists a sufficiently small neighborhood $\mathscr{U}_{x}$ of the point $x$ satisfying that $(2 \lambda-\delta)(y) \neq 0$ for each $y \in \mathscr{U}_{x}$. This, combined with Lemma $\mathrm{A}(2)$ and (3.1), yields the following equation on the neighborhood $\mathscr{U}_{x}$ :

$$
\lambda+\frac{\delta \lambda+\frac{c}{2}}{2 \lambda-\delta}=\frac{2 \sqrt{|c|}}{\sqrt{3}} .
$$

Thus, from the constancy of $\delta$ and this equation we find that $\lambda$ is constant on $\mathscr{U}_{x}$.

We finally consider the case of $2 \lambda-\delta=0$ at some point of $M$. We shall verify that $2 \lambda-\delta$ vanishes identically on $M$. Assume that $2 \lambda-\delta \neq 0$ at some point $x_{0} \in M$, and set $y_{0}=(2 \lambda-\delta)\left(x_{0}\right)$. Let $N$ be the subset of those points $x \in M$ such that $(2 \lambda-\delta)(x)=y_{0}$. Clearly $N$ is a non-empty closed subset of $M$. It is also open, since the discussion in the case of $2 \lambda-\delta \neq 0$ means that the function $2 \lambda-\delta$ is constantly equal to $y_{0} \neq 0$ on some neighborhood of each point $x \in N$. Since $M$ is connected, we find that $N=M$, which is a contradiction. So we find that $\lambda=\delta / 2$ on $M$.

Thus we can see that our real hypersurface is a Hopf hypersurface with constant principal curvatures. In consideration of Theorem $\mathrm{A}$ we see that $M$ is of type either $\left(A_{0}\right),\left(A_{1}\right),\left(A_{2}\right)$ or $(B)$. We shall check $\left.2_{a}\right)$ for these real hypersurfaces one by one. For the principal curvatures of real hypersurfaces of type (A) and their properties, see [9]. 
Let $M$ be of type $\left(\mathrm{A}_{0}\right)$. Then $A X=(\sqrt{|c|} / 2) X$ for all $X \in T^{0} M$ and $A \xi=\sqrt{|c|} \xi$, which implies $(\phi A+A \phi) X=\sqrt{|c|} \phi X$ for all $X \in T M$. So we have $d \eta(X, Y)=(-\sqrt{|c|} / 2) g(X, \phi Y)$ for all $X, Y \in T M$, which is a contradiction. Thus we can see that this real hypersurface does not satisfy $2_{a}$ ).

Let $M$ be of type $\left(\mathrm{A}_{1,0}\right)$. Then $A X=(\sqrt{|c|} / 2) \operatorname{coth}(\sqrt{|c|} r / 2) X$ for all $X \in T^{0} M$ and $A \xi=\sqrt{|c|} \operatorname{coth}(\sqrt{|c|} r) \xi$. Hence, by the same computation as above $d \eta(X, Y)=(-\sqrt{|c|} / 2) \operatorname{coth}(\sqrt{|c|} r / 2) g(X, \phi Y)$. Thus we obtain the equation $\sqrt{|c|} \operatorname{coth}(\sqrt{|c|} r / 2)=2 \sqrt{|c|} / \sqrt{3}$. Solving this equation, we know that the radius $r$ of real hypersurfaces of type $\left(\mathrm{A}_{1,0}\right)$ is expressed as $r=(2 / \sqrt{|c|}) \log _{e}(2+\sqrt{3})$. Then this real hypersurface satisfies $\left.2_{a}\right)$.

Let $M$ be of type $\left(\mathrm{A}_{1,1}\right)$. Then $A X=(\sqrt{|c|} / 2) \tanh (\sqrt{|c|} r / 2) X$ for all $X \in T^{0} M$ and $A \xi=\sqrt{|c|} \operatorname{coth}(\sqrt{|c|} r) \xi$. Hence we get $d \eta(X, Y)=(-\sqrt{|c|} / 2)$. $\tanh (\sqrt{|c|} r / 2) g(X, \phi Y)$, so that $\sqrt{|c|} \tanh (\sqrt{|c|} r / 2)=2 \sqrt{|c|} / \sqrt{3}$, which is a contradiction. Thus this real hypersurface does not satisfy Condition $2_{a}$ ).

Let $M$ be of type $\left(\mathrm{A}_{2}\right)$. Then the holomorphic distribution $T^{0} M$ of $M$ is decomposed as $T^{0} M=V_{\lambda_{1}}^{0} \oplus V_{\lambda_{2}}^{0}=V_{\lambda_{1}} \oplus V_{\lambda_{2}}$ with $\lambda_{1}=(\sqrt{|c|}) \operatorname{coth}(\sqrt{|c|} r / 2)$, $\lambda_{2}=(\sqrt{|c|}) \tanh (\sqrt{|c|} r / 2)$ and $A \xi=\sqrt{|c|} \operatorname{coth}(\sqrt{|c|} r) \xi$. Note that $\phi V_{\lambda_{1}}^{0}=V_{\lambda_{1}}^{0}$ and $\phi V_{\lambda_{2}}^{0}=V_{\lambda_{2}}^{0}$. These equalities imply that Equation (3.1) does not hold. Hence this real hypersurface does not hold $2_{a}$ ).

Let $M$ be of type (B). Then it follows from (3.1) that

$$
\frac{\sqrt{|c|}}{2} \operatorname{coth}\left(\frac{\sqrt{|c|} r}{2}\right)+\frac{\sqrt{|c|}}{2} \tanh \left(\frac{\sqrt{|c| r}}{2}\right)=\frac{2 \sqrt{|c|}}{\sqrt{3}} .
$$

Solving this equation, we get $r=(1 / \sqrt{|c|}) \log _{e}(2+\sqrt{3})$, so that $M$ has two distinct principal curvatures $\lambda_{1}=\delta=\sqrt{3|c|} / 2$ and $\lambda_{2}=\sqrt{|c|} /(2 \sqrt{3})$, which yields that this real hypersurface satisfies $2_{a}$ ). Hence our discussion asserts that a real hypersurface of $\mathrm{CH}^{n}(c)$ satisfies $2_{a}$ ) if and only if $M$ is locally congruent to either the geodesic sphere $G(r)$ of radius $r=(2 / \sqrt{|c|}) \log _{e}(2+\sqrt{3})$ (i.e., $(\sqrt{|c|} / 2) \operatorname{coth}(\sqrt{|c|} r / 2)=\sqrt{|c|} / \sqrt{3})$ or the real hypersurface of type (B) with two distinct principal curvatures. However the former case does not satisfy $2_{b}$ ). Indeed, every geodesic $\gamma=\gamma(s)$ on $G(r)$ of radius $r=(2 / \sqrt{|c|}) \log _{e}(2+\sqrt{3})$ with initial vector $\dot{\gamma}(0)$ orthogonal to $\xi_{\gamma(0)}$ is mapped to a circle of the same curvature $\sqrt{|c|} / \sqrt{3}$ (cf. [5]). Therefore we can see that Condition (2) implies Condition (1).

We finally suppose Condition (3). We shall study on the open dense subset $\mathscr{U}$ of $M$, which is given by Remark 3. Condition $3_{a}$ ) implies that our real hypersurface $M$ is a Hopf hypersurface. Next, for each vector $X, Y$ of any restricted principal distribution $V_{\lambda_{i}}^{0}$, from Condition $3_{b}$ ) we get

$$
\begin{aligned}
0 & =\left(\nabla_{X} A\right) Y=\nabla_{X}(A Y)-A \nabla_{X} Y \\
& =\nabla_{X}\left(\lambda_{i} Y\right)-A \nabla_{X} Y \\
& =\left(X \lambda_{i}\right) Y+\left(\lambda_{i} I-A\right) \nabla_{X} Y,
\end{aligned}
$$


so that

$$
\left(X \lambda_{i}\right) Y+\left(\lambda_{i} I-A\right) \nabla_{X} Y=0 .
$$

We here take an arbitrary vector $Z \in V_{\lambda_{j}}^{0}$ with $\lambda_{i} \neq \lambda_{j}$.

Note that there exists such a vector $Z$. To do show that, we assume that there does not exist such $Z$. Then there exists an open set $\mathscr{V}$ of $M$ such that $T^{0} \mathscr{V}=V_{\lambda_{i}}^{0}$, which means that $A v=\lambda_{i} v$ for any $v \in T^{0} \mathscr{V}$, i.e., our real hypersurface $\mathscr{V}^{\lambda}$ is totally $\eta$-umbilic in the ambient space $\mathbf{C} H^{n}(c)$. Hence this real hypersurface $\mathscr{V}$ is of type (A). So the shape operator $A$ of $\mathscr{V}$ satisfies the following differential equation:

$$
\left(\nabla_{X} A\right) Y=(-c / 4)(g(\phi X, Y) \xi+\eta(Y) \phi X)
$$

for all $X, Y \in T \mathscr{V}$ (see [9]). Then for any unit vector $X$ orthogonal to $\xi$, from the above equation we have $\left(\nabla_{X} A\right) \phi X=(-c / 4) \xi \neq 0$, which contradicts to Condition $3_{b}$ ).

Then, taking the inner product of (3.2) and the vector $Z \in V_{\lambda_{j}}^{0}$ with $\lambda_{i} \neq \lambda_{j}$, we obtain

$$
0=g\left(\left(\lambda_{i} I-A\right) \nabla_{X} Y, Z\right)=\left(\lambda_{i}-\lambda_{j}\right) g\left(\nabla_{X} Y, Z\right),
$$

so that $g\left(\nabla_{X} Y, Z\right)=0$, which shows that

$$
\nabla_{X} Y \in V_{\lambda_{i}}^{0} \oplus\{\xi\}_{\mathbf{R}} \text { for each } X, Y \in V_{\lambda_{i}}^{0} .
$$

We shall verify that $g\left(\nabla_{X} Y, \xi\right)=0$. It follows from (2.5) that

$$
g\left(\nabla_{X} Y, \xi\right)=-g\left(Y, \nabla_{X} \xi\right)=-g(Y, \phi A X)=-\lambda_{i} g(Y, \phi X) .
$$

On the other hand, from (2.6) and Condition $\left.3_{b}\right)$ we find that $g(\phi X, Y)=0$ for each $X, Y \in V_{\lambda_{i}}^{0}$. This, together with (3.3) and (3.4), yields that $\nabla_{X} Y \in V_{\lambda_{i}}^{0}$ for every vector $X, Y$ of any restricted principal distribution $V_{\lambda_{i}}^{0}$. Hence our real hypersurface $M$ is of type (B) on the open dense subset $\mathscr{U}$ (see Lemma C), which implies that $M$ is globally of type (B).

The rest of the proof is to determine real hypersurfaces of type (B) satisfying Condition $3_{c}$ ).

To do this, we review the following fact. We take a geodesic $\gamma=\gamma(s)$ on a hypersurface $M^{n}$ isometrically immersed into an $(n+1)$-dimensional Riemannian manifold $\tilde{M}^{n+1}$ (with Riemannian metric $g$ ). Suppose that the geodesic $\gamma$ is mapped to a circle of positive curvature $k$ in the ambient space $\tilde{M}^{n+1}$. Then the shape operator $A$ of $M^{n}$ in $\tilde{M}^{n+1}$ satisfies

$$
A \dot{\gamma}(s)=k \dot{\gamma}(s) \text { for each } s \text { or } A \dot{\gamma}(s)=-k \dot{\gamma}(s) \text { for each } s .
$$

In fact, it follows from (2.1), (2.2) and (2.7) that $g(A \dot{\gamma}, \dot{\gamma}) A \dot{\gamma}=k^{2} \dot{\gamma}$ holds on the curve $\gamma$. This, combined with $k \neq 0$, yields (3.5). 
In view of the above fact and Condition $3_{c}$ ) we have only to consider the following equation

$$
\frac{\sqrt{|c|}}{2} \operatorname{coth}\left(\frac{\sqrt{|c|} r}{2}\right)=3 \frac{\sqrt{|c|}}{2} \tanh \left(\frac{\sqrt{|c| r}}{2}\right) .
$$

Solving this equation, we see that $r=(1 / \sqrt{|c|}) \log _{e}(2+\sqrt{3})$. Thus we obtain Condition (1).

Remark 2. As an immediate consequence of the discussion in the proof of our Theorem we have the following:

Corollary. A connected real hypersurface $M$ of $\mathbf{C H}(c), n \geqq 2$ is of type (B) if and only if $M$ satisfies the following two conditions:

(1) The holomorphic distribution $T^{0} M$ of $M$ is decomposed as the direct sum of restricted principal distributions $V_{\lambda_{i}}^{0}=\left\{X \in T^{0} M \mid A X=\lambda_{i} X\right\}$;

(2) The derivative of the shape operator $A$ of $M$ satisfies $\left(\nabla_{X} A\right) Y=0$ for all vectors $X, Y$ of each $V_{\lambda_{i}}^{0}$ in (1).

Remark 3. As a matter of fact, if a real hypersurface $M$ of $\mathbf{C} H^{n}(c)$ satisfies Condition (1) in the above Corollary, then $M$ is a Hopf hypersurface. Note that the converse does not hold in general. However every Hopf hypersurface $M^{2 n-1}$ of $\mathbf{C H}^{n}(c)$ satisfies locally Condition (1) on an open dense subset $\mathscr{U}=\left\{\begin{array}{l|l}x \in M^{2 n-1} & \begin{array}{l}\text { the multiplicity of every principal curvature of } M^{2 n-1} \text { in } \\ \mathbf{C} H^{n}(c) \text { is constant on some neighborhood } \mathscr{V}_{x}(\subset \mathscr{U}) \text { of } x\end{array}\end{array}\right\}$ of $M^{2 n-1}$.

Remark 4. If we remove Condition $2_{b}$ ), our Theorem is not true. In fact, the geodesic sphere $G(r)$ with radius $r=(2 / \sqrt{|c|}) \log _{e}(2+\sqrt{3})$ in $\mathbf{C} H^{n}(c)$ satisfies Condition $22_{a}$ ).

\section{REFERENCES}

[1] T. Adachi, M. Kameda And S. MaedA, Real hypersurfaces which are contact in a nonflat complex space form, Hokkaido Math. J. 40 (2011), 205-217.

[2] T. Adachi And S. Maeda, Global behaviours of circles in a complex hyperbolic space, Tsukuba J. Math. 21 (1997), 29-42.

[3] J. BERndT, Real hypersurfaces with constant principal curvatures in complex hyperbolic space, J. Reine Angew. Math. 395 (1989), 132-141.

[4] J. Berndt AND H. TAMARU, Cohomogeneity one actions on noncompact symmetric spaces of rank one, Trans. Amer. Math. Soc. 359 (2007), 3425-3438.

[ 5 ] B. Y. Chen AND S. MAEda, Hopf hypersurfaces with constant principal curvatures in complex projective or complex hyperbolic spaces, Tokyo J. Math. 24 (2001), 133-152.

[6] S. MaEda, A charactrization of the homogeneous real hypersurface of type (B) with two distinct constant principal curvatures in a complex hyperbolic space, Sci. Math. Japonicae 68 (2008), 1-10. 
[7] Y. Maeda, On real hypersurfaces of a complex projective space, J. Math. Soc. Japan 28 (1976), 529-540.

[ 8 ] S. Montiel, Real hypersurfaces of a complex hyperbolic space, J. Math. Soc. Japan 37 (1985), 515-535.

[9] R. Niebergall and P. J. Ryan, Real hypersurfaces in complex space forms, Tight and taut submanifolds (T. E. Cecil and S. S. Chern, eds.), Cambridge University Press, 1998, 233-305.

[10] K. OKumura AND S. MaEda, Three real hypersurfaces some of whose geodesics are mapped to circles with the same curvature in a nonflat complex space form, Geom. Dedicata 156 (2012), 71-80.

[11] R. TAKAGI, On homogeneous real hypersurfaces in a complex projective space, Osaka $\mathbf{J}$. Math. 10 (1973), 495-506.

Katsufumi Yamashita

DePartment of Mathematics

SAGA UNIVERSITY

1 Honzyo, SAGa 840-8502

JAPAN

E-mail: ky-karatsucity@sgr.bbiq.jp

Sadahiro Maeda

Department of Mathematics

SAGA UniVERSITY

1 Honzyo, Saga 840-8502

JAPAN

E-mail: smaeda@ms.saga-u.ac.jp 